\title{
Sobrecarga de trabalho da Enfermagem e incidentes e eventos adversos em pacientes internados em UTI
}

\author{
Nursing workload and occurrence of incidents and adverse events in ICU patients
}

Sobrecarga de trabajo de Enfermería y los incidentes y eventos adversos en pacientes hospitalizados en la UCI

\section{Marcia Cristina Zago Novaretti', Edzangela de Vasconcelos Santos', Ligia Maria Quitério', Renata Mahfuz Daud-Gallotti'}

\author{
' Universidade Nove de Julho, Programa de Pós-Graduação em Gestão em Sistemas de Saúde. São Paulo-SP, Brasil.
}

\author{
Submissão: 29-05-2013 Aprovação: 08-07-2014
}

\section{RESUMO}

Estudo prospectivo, tipo coorte que visou identificar a influência da sobrecarga de trabalho da Enfermagem na ocorrência de incidentes sem lesão e eventos adversos em 399 pacientes internados em Unidades de Terapia Intensiva (UTI). Para coleta de dados, foi aplicado um questionário estruturado e feita análise de prontuários. Nessas admissões, aproximadamente 78\% dos incidentes sem lesão e eventos adversos em pacientes foram relacionados à esfera da Enfermagem. Essas ocorrências foram atribuídas à sobrecarga de trabalho, aumentaram o número de dias de internação e o risco de óbito dos pacientes estudados. É fundamental que os gerentes de enfermagem atuem no processo de gestão de pessoas no âmbito hospitalar, evitando a sobrecarga de trabalho e proporcionando, consequentemente, aumento da segurança do paciente.

Descritores: Enfermagem; Saúde; Gestão; Segurança do Paciente; Trabalho.

\begin{abstract}
This prospective cohort study aimed to identify the influence of nursing work overload on the occurrence of incidents without injury and adverse events in 399 patients hospitalized in Intensive Care Units (ICU). For data collection, a structured questionnaire was administered and an analysis of medical records was performed. In these admissions, approximately $78 \%$ of incidents without injury and adverse events in patients were related to the sphere of Nursing. These occurrences were attributed to overwork, increased the number of days of hospitalization and the risk of death of patients. It is essential that nursing managers work on the staff hospital management avoiding work overload to contribute for patient safety.
\end{abstract}

Key words: Nursing; Health Care; Management; Patient Safety; Job.

\section{RESUMEN}

Estudio de cohorte prospectivo que tuvo como objetivo identificar la influencia de la carga de trabajo de enfermería en la ocurrencia de incidentes sin lesión y los eventos adversos en 399 pacientes ingresados en Unidades de Cuidados Intensivos $(\mathrm{UCl})$. Para la recolección de datos, se administró un cuestionario estructurado y realizó el análisis de los registros en prontuarios. En estos ingresos, aproximadamente el 78\% de los incidentes sin lesión y los eventos adversos en los pacientes estaban relacionados con el ámbito de la Enfermería. Estos hechos fueron atribuidos al exceso de trabajo, aumentaran el número de días de hospitalización y el riesgo de muerte de los pacientes. Es esencial que los responsables de enfermería que actúan en el proceso de gestión de las personas en los hospitales, eviten el exceso de trabajo y por lo tanto proporcionar una mayor seguridad de los pacientes.

Palabras clave: Enfermería; Salud; Gestión; Seguridad del Paciente; Trabajo. 


\section{INTRODUÇÃO}

Um dos maiores desafios na gestão em saúde é a análise dos indicadores de qualidade e o impacto potencial na segurança do paciente. A segurança do paciente tem por objetivo a redução do risco de danos desnecessários relacionados aos cuidados de saúde para um mínimo aceitável, de acordo com conhecimento atual, dos recursos disponíveis e no contexto em que os cuidados foram prestados ${ }^{(1)}$. As falhas na segurança do paciente causam também sofrimento humano e aumento de custos $^{(2)}$. Dentre os diversos fatores que podem influenciar na segurança de pacientes internados, destacam-se os incidentes e eventos adversos $(E A)^{(3)}$. Incidentes de segurança do paciente, conforme classificação da Organização Mundial de Saúde, são eventos ou circunstâncias que poderiam resultar, ou resultam em complicações desnecessárias ao paciente decorrentes do cuidado à saúde ${ }^{(1)}$. Entende-se por "desnecessária" neste contexto, os erros, transgressões e atos deliberadamente perigosos que ocorrem em cuidados de saúde. Os incidentes surgem quer de atos intencionais ou não intencionais. Os erros são geralmente não intencionais, enquanto que as transgressões são usualmente intencionais. Um incidente pode ser classificado em incidente sem danos ou com danos. Este último é também chamado de evento adverso (EA). Dano implica em prejuízo na estrutura ou nas funções do corpo e/ou qualquer efeito deletério daí resultante, incluindo lesão, doença, sofrimento, incapacidade ou morte, e pode ser físico, social ou psicológico. Os eventos adversos e incidentes são relevantes indicadores de qualidade, fornecendo informações imprescindíveis para o planejamento de um sistema de saúde mais seguro ${ }^{(4)}$.

Embora os EAs possam acontecer em qualquer setor da assistência à saúde, pacientes em Unidades de Terapia Intensiva (UTI) são particularmente vulneráveis a essas complicações, quer seja pela necessidade de decisões de alto risco, muitas vezes de forma urgente, a utilização de arsenal medicamentoso mais complexo, um número elevado de procedimento diagnósticos, pelo alto número de intervenções realizadas, em função da gravidade e labilidade dos pacientes internados nesse local(5). Apesar dessa vulnerabilidade, são poucas publicações relacionadas à segurança de pacientes submetidos a cuidados intensivos.

A frequência de eventos adversos (EA) em pacientes hospitalizados varia amplamente, de 10 a $60 \%{ }^{(6-7)}$, enquanto que os incidentes sem lesão podem chegar a impressionantes $60 \%$ das internações. Em uma UTI, aproximadamente $20 \%$ dos pacientes podem sofrer um EA e, destes cerca de $40-45 \%$ podem ser considerados evitáveis ${ }^{(5,8)}$ Interessante notar que, em quase metade dos episódios relatados, as complicações são consideradas preveníveis ou evitáveis ${ }^{(1)}$. Estudos norte-americanos mostram que os EAs evitáveis contribuíram para a morte de 44.000 a 98.000 pessoas/ano ${ }^{(9)}$. Além disso, os EAs afetam as taxas de sobrevida dos pacientes significativamente e independentemente ${ }^{(10)}$.

$\mathrm{Na}$ análise de segurança dos pacientes em cuidados intensivos, destaca-se a questão da deteç̧ão de incidentes sem lesão (I), uma vez que estes podem ser seguidos de eventos adversos e comprometer a segurança dos pacientes. A identificação de padrões recorrentes e de fatores que possam contribuir para os incidentes sem lesão pode ser considerada um pré-requisito para a elaboração de estratégias preventivas efetivas nesse quesito. Pesquisas sobre incidentes sem lesão sem segurança do paciente são escassas ${ }^{(11)}$.

$\mathrm{Na}$ análise dos fatores relacionados aos EA em pacientes em UTI, destaca-se o papel da Enfermagem, uma vez que este é o local em que há maior demanda para as atividades dos enfermeiros e há exigência de ações rápidas e de observação continuada por parte desses profissionais ${ }^{(4)}$. As condições de trabalho do pessoal de enfermagem, caracterizadas, por vezes, pela sobrecarga de trabalho e pela jornada em regime de plantões são fatores de risco para a segurança do paciente ${ }^{(12)}$. Carayon \& Gurses ${ }^{(13)}$ já referiam, em 2005, que a inadequação do quadro de enfermagem é fator que compromete a qualidade do cuidado de pacientes em cuidados intensivos. Weissman et al. ${ }^{(14)}$ inclusive observaram que um aumento de $0,1 \%$ na razão paciente/enfermeiro levou a um aumento de 28,0\% na taxa de eventos adversos nas instituições pesquisadas. Esses autores também demonstraram que a carga de trabalho é um fator de risco para a segurança dos pacientes em UTI.

Embora a carga de trabalho da Enfermagem tenha sido evidenciada como fator de risco para a segurança do paciente, em especial naqueles em UTI, a grande maioria dos estudos realizados é do tipo retrospectivo e, por isso, compromete a detecção e o registro de EAs, limitando consideravelmente a reprodutibilidade das conclusões observadas. Assim, a presente pesquisa foi desenvolvida com o objetivo de estudar, prospectivamente, a influência da carga de trabalho da Enfermagem no risco de incidentes sem lesão e de eventos adversos relacionados à competência de enfermagem em pacientes internados em unidades de terapia intensiva.

\section{METODOLOGIA}

Trata-se de estudo observacional, prospectivo, tipo coorte, qualitativo, descritivo realizado em dois hospitais universitários de alta complexidade, o Instituto Central do Hospital das Clínicas da Faculdade de Medicina da Universidade de São Paulo (HC-FMUSP) que conta com 910 leitos e o Hospital Universitário da Universidade de São Paulo (HU) que tem 258 leitos, localizados no município de São Paulo. O projeto de pesquisa foi submetido à análise dos Comitês de Ética em Pesquisa das instituições participantes onde obteve aprovação (Cappesq 0279/07).

Os cenários foram Unidades de Terapia Intensiva dos hospitais participantes, sendo 27 leitos do HC-FMUSP e 20 leitos do HU. As UTIs participantes do HC-FMUSP apresentam alta demanda pelo fato deste hospital ser referência para outros hospitais de alta complexidade na sua área de abrangência. A UTI do HU atende a pacientes clínico-cirúrgicos.

Foram incluídos neste estudo todos os pacientes com idade superior a 15 anos internados nos serviços descritos acima, no período de maio a agosto de 2009. Os pacientes foram acompanhados até sua saída dos serviços incluídos no estudo (alta ou óbito).

Os EAs e incidentes sem lesão que tiveram seu início antes da admissão dos pacientes nos serviços estudados não foram incluídos. 
Os dados foram produzidos por meio de preenchimento de formulários específicos para o registro de possíveis EAs/I a partir da leitura e análise diárias dos prontuários, bem como aqueles notificados espontaneamente à equipe de pesquisa. As visitas diárias nas respectivas UTIs com a equipe multiprofissional foram acompanhadas por observador deste estudo. Os EAs e incidentes foram também identificados por minuciosa revisão dos prontuários médicos dos pacientes incluídos. Neste processo, revisores previamente treinados, analisaram detalhadamente todas as partes que compõem o prontuário, incluindo evoluções e prescrições médicas; solicitações e relatórios de interconsultas; evoluções, prescrições, anotações e controles de enfermagem. Uma busca minuciosa por problemas relacionados à coleta e encaminhamento de exames foi realizada nos laboratórios das instituições envolvidas.

Os incidentes sem lesão e os EAs foram classificados conforme a sua natureza: se falhas administrativas, médicas, de enfermagem, cirúrgicas, relacionadas a medicamentos e à infeção hospitalar ${ }^{(1)}$. Neste estudo nos restringimos a apresentação dos dados referentes à competência de enfermagem. Os pesquisadores responsáveis categorizaram as observações encontradas em EA/I relacionados à competência de enfermagem, de acordo com o relatório técnico da Organização Mundial da Saúde publicado em 2011, conforme o tipo ${ }^{(1)}$.

Incidentes sem lesão (I): falhas no seguimento da prescrição de enfermagem, falhas no seguimento da prescrição médica não medicamentosa pela equipe de enfermagem, falhas na administração de medicamentos, problemas da Enfermagem a manipulação de sondas e cateteres, problemas no encaminhamento de exames, falhas no registro de enfermagem, problemas na administração de dietas enterais e exames não realizados.

Eventos Adversos (EAs): relacionados a medicamentos, dermatites, assaduras, úlceras por pressão, falhas no diagnóstico e tratamento médicos, flebites, problemas de contenção de pacientes.

Também foram registradas as informações relacionadas aos diagnósticos de admissão nas UTIs; comorbidades por meio da escala de Charlson ${ }^{(15)}$; tempo de permanência nos serviços; gravidade clínica à admissão nas UTIs por APACHEII e SAPII ${ }^{(16)}$ e a condição de saída das UTIs e do Hospital.

A carga de trabalho de enfermagem foi mensurada pelo índice Nursing Activities Score (NAS). Esse instrumento foi desenvolvido por Miranda et al. (2003) ${ }^{(17)}$ e traduzido e validado por Queijo e Padilha ${ }^{(18)}$; consiste de 23 itens relacionados às intervenções médicas e de enfermagem a que o paciente foi submetido na UTI. O cálculo do escore total do NAS resulta do somatório dos itens pontuados que expressa em porcentagem o tempo gasto pela equipe de enfermagem na assistência ao doente em UTI. Um formulário NAS foi preenchido por cada profissional de enfermagem diretamente envolvido no cuidado de cada paciente no final de cada turno de trabalho. O NAS diário foi determinado para cada paciente durante o período de acompanhamento com o intuito de estimar o NAS médio para cada paciente no período. Considerando que, na UTI, dois leitos, no mínimo, foram atribuídos para cada profissional de enfermagem, foi considerado que um NAS $\geq 51 \%$ era indicativo de uma sobrecarga de trabalho durante o período de estudo ${ }^{(19)}$.
As informações coletadas foram transferidas para uma base de dados específica por dupla digitação. As variáveis contínuas foram analisadas pelos testes estatísticos descritivos (média, desvio padrão, mediana, valor máximo e valor mínimo e suas médias). As variáveis categóricas foram apresentadas com frequências absolutas e relativas. Foram analisadas as correlações entre EAs/incidentes sem lesão e tempo de permanência, carga de trabalho de enfermagem e índices de gravidade dos pacientes pelo teste de Spearman. Os fatores que mostraram resultados estatisticamente significativos na análise univariada foram selecionados para análise multivariada. Modelos de regressão logística foram aplicados, estimando-se "odds ratios" e respectivos intervalos de confiança. Os programas estatísticos utilizados foram STATA versão 10.0 (Stata Corp, College Station TX) e SPSS (Statistical Package for the Social Sciences), versão 19.0. Foram considerados resultados significantes quando $p<0,05$.

\section{RESULTADOS}

Um total de 399 admissões referentes a 380 pacientes foram estudadas, sendo que 79 pacientes (20,8\% dos pacientes) evoluíram para óbito durante a internação nas UTIs pesquisadas. As admissões realizadas no HC-FMUSP apresentaram maior complexidade e foram mais prolongadas, justificada pelo maior número de casos complexos internados nesse hospital quando comparadas com aquelas feitas no HU (Tabela 1).

A carga de trabalho da Enfermagem mensurada pelo NAS foi maior no HC-FMUSP $(69,55 \pm 1,40)$ quando comparado ao $\mathrm{HU}(61,97 \pm 2,25)$. Tendo em vista que cada profissional de enfermagem assistiu a, no mínimo dois pacientes, constata-se a existência de sobrecarga da equipe de enfermagem, uma vez que os índices NAS observados nos dois hospitais estudados superam em muito o valor acima do qual é considerado sobrecarga de trabalho (NAS $\geq 51 \%$ ).

Um total de 15.054 EAs e incidentes sem lesão foram detectados no período estudado, afetando 394 das 399 (98,75\%) das internações analisadas. Os incidentes sem lesão (12.737, 84,6\%) acometeram 391 admissões e os EAs (2.317, 15,4\%) atingiram 296 admissões. Desse modo, 74,2\% das admissões sofreram ao menos um EA durante a permanência nas UTIs avaliadas. Ao compararmos o grupo de admissões que sofreu pelo menos um EA com aquele que não sofreu EAs, observamos que, no primeiro grupo, a gravidade clínica na admissão à UTI (Apachell) foi maior (15,03 \pm 0,44 nas internações com EAs e 10,59 $\pm 0,68$ nas sem EAs, $p<0,001)$, as admissões foram mais prolongadas $(11,08$ dias $\pm 0,63$ nas internações com EAs e 2,19 dias $\pm 0,18$ nas sem EAs, $p<0,001)$. Destaca-se a média da carga de trabalho da equipe de enfermagem que foi mais elevada nos pacientes com EAs, NAS (\%) 66,2 \pm 1,05 que naqueles sem EA, 60,25 $\pm 1,85,(p=0,02)$. A análise bivariada mostrou uma forte e significativa correlação do número de EAs com o tempo de internação nas UTIs $(0,800$; $\mathrm{p}<0,0001)$. A gravidade clínica (pontuação Apache II) na admissão à UTI $(0,35 ; p<0,0001)$, a carga de trabalho da Enfermagem (NAS), 0,191 e $p<0,0001$ e o peso da comorbidades (pontuação escala de Charlson), 0,128 e p<0,0001 apresentaram uma correlação significativa, com a ocorrência de EAs. 
Tabela 1 - Distribuição das características tempo de internação e condições clínicas de entrada, condições de saída, gravidade do paciente de acordo com o hospital estudado, São Paulo-SP, 2009

\begin{tabular}{|c|c|c|c|}
\hline Variável & $\begin{array}{r}\text { HC-FMUSP } \\
n=202\end{array}$ & $\begin{array}{r}\mathrm{HU} \\
\mathrm{n}=197\end{array}$ & $\mathrm{p}$ \\
\hline $\begin{array}{l}\text { Tempo de Internação UTI (dias) } \\
\text { Média } \pm \text { EP (MIN-MAX) }\end{array}$ & $\begin{array}{r}10,47 \pm 0,77 \\
(1,0-70,0)\end{array}$ & $\begin{array}{r}7,10 \pm 0,65 \\
(1,0-49,0)\end{array}$ & 0,01 \\
\hline $\begin{array}{l}\text { Tempo de Internação no hospital (dias) } \\
\text { Média } \pm \text { EP (MIN-MAX) }\end{array}$ & $\begin{array}{r}19,78 \pm 1,20 \\
(1,0-84,0)\end{array}$ & $\begin{array}{r}13,62 \pm 1,01 \\
(1,0-95,0)\end{array}$ & $<0,01$ \\
\hline $\begin{array}{l}\text { Gravidade à Admissão APACHE (pontos) } \\
\text { Média + EP (MIN-MAX) }\end{array}$ & $\begin{array}{r}16,04 \pm 0,50 \\
(0-48,0)\end{array}$ & $\begin{array}{r}11,68 \pm 0,54 \\
(0-40,0)\end{array}$ & $<0,01$ \\
\hline $\begin{array}{l}\text { NAS }(\%) \\
\text { Média + EP (MIN-MAX) }\end{array}$ & $\begin{array}{r}69,55 \pm 1,40 \\
(19,55-145,56)\end{array}$ & $\begin{array}{r}61,97 \pm 2,25 \\
(9,88-114,4)\end{array}$ & $<0,01$ \\
\hline Condição de saída das UTIs (número pacientes; \%) Alta / Óbito & $155(79,5 \%) / 40(20,5 \%)$ & $154(83,25 \%) / 31(16,75 \%)^{* *}$ & \\
\hline Condição de saída do Hospital (número pacientes; \%) Alta: Óbito & $146(74,9 \%): 49(25,1 \%)^{*}$ & $139(75,14 \%): 46(24,86 \%)^{* *}$ & \\
\hline
\end{tabular}

n: número de admissões; erro padrão;

MIN: valor mínimo apresentado;

MAX: valor máximo apresentado

*: resultados se referem a 195 pacientes

**: resultados se referem a 185 pacientes

Tabelas 2 - Incidentes sem lesão relacionados à esfera da Enfermagem nas admissões nas UTIs estudadas, São Paulo-SP, 2009

\begin{tabular}{lcc}
\hline Tipos de incidentes sem lesão & n & \multicolumn{1}{c}{ \% de incidentes } \\
\hline Falhas no seguimento da prescrição de enfermagem & 5610 & 55,46 \\
Falhas no preparo e administração de medicações & 1563 & 15,45 \\
Problemas da Enfermagem a manipulação de sondas e cateteres & 892 & 744 \\
Problemas na coleta e no encaminhamento de exames & 577 & 7,35 \\
Falhas no registro de dados nos prontuários pela Enfermagem & 507 & 5,70 \\
Problemas na administração de dietas enterais & 222 & 5,01 \\
Falhas no seguimento da prescrição médica não medicamentosa pela Enfermagem & 10.115 & 2,19 \\
TOTAL & & 100 \\
\hline
\end{tabular}

Tabela 3 - Eventos adversos relacionados à esfera da Enfermagem nas admissões nas UTIs estudadas, São Paulo-SP, 2009

\begin{tabular}{lcc}
\hline Tipos de eventos adversos & n & \% de incidentes \\
\hline Dermatites, assaduras, úlceras por pressão & 376 & 60,45 \\
Problemas na contenção física de pacientes & 113 & 18,17 \\
Flebite & 72 & 11,58 \\
Falhas da enfermagem na manipulação de cateteres e sondas & 57 & 9,16 \\
Quedas de pacientes & 4 & 0,64 \\
TOTAL & 622 & 100 \\
\hline
\end{tabular}

Os incidentes sem lesão relacionados à competência da enfermagem, foco deste estudo, totalizaram 10.115, ou seja $79,4 \%$ do total de incidentes sem lesão. As falhas no seguimento da prescrição da Enfermagem (5.610, 55,46\%) foram os incidentes mais frequentemente observados neste estudo (Tabela 2). Os eventos adversos associados à atuação da Enfermagem, também objetivo deste estudo, corresponderam a $622(26,80 \%)$ são apresentados na Tabela 3. O tipo de EA relacionado à esfera da Enfermagem mais comumente detectado neste estudo foram as dermatites, assaduras e úlceras de pressão, que são complicações passíveis de prevenção pelos cuidados adequados de enfermagem.

A análise bivariada mostra uma forte correlação entre tempo de internação $(0,825 ; p<0,0001)$ e sobrecarga de trabalho de enfermagem $(0,331 ; p<0,0001)$ e os incidentes sem lesão e EAs relacionados à enfermagem. Correlações significativas, embora menos intensas, também foram encontradas quanto ao número de incidentes sem lesão e EAs de enfermagem e a gravidade clínica à admissão, $(0,220$; $p=0,002)$, Tabela 4 . 
Tabela 4 - Correlação bivariada de ocorrências de Incidentes sem lesão e eventos adversos e tempo de internação nas UTIs, APACHE, e NAS

\begin{tabular}{lccc}
\hline $\begin{array}{l}\text { Variável } \\
\text { (Spearman) }\end{array}$ & $\begin{array}{c}\text { Dias de internação } \\
\text { em UTI }\end{array}$ & $\begin{array}{c}\text { NAS } \\
\text { (\%) }\end{array}$ & $\begin{array}{c}\text { APACHE II } \\
\text { (pontos) }\end{array}$ \\
\hline Incidentes sem lesão + Eventos Adversos & 0,825 & 0,331 & 0,220 \\
& $\mathrm{p}<0,0001$ & $\mathrm{p}<0,0001$ & $\mathrm{p}<0,002$ \\
\hline
\end{tabular}

A regressão logística univariada para a ocorrência de pelo menos um EA relacionado à enfermagem mostrou que o tempo de internação superior a 3 dias foi o fator de risco isolado mais importante $(\mathrm{OR}$ simples $=11,44$; IC 95\%=6,81-19,22; $p<0,001)$. As variáveis sinalizadoras de gravidade também se associaram de forma isolada à ocorrência de ao menos um EA de enfermagem (OR simples =2,46; IC 95\% = 1,63$3,74 ; p<0,001)$. Outro fator de risco isolado significativo foi a sobrecarga de trabalho de enfermagem (OR simples $=2,81$; IC 95\%=1,67-4,72; $p<0,001)$.

$\mathrm{Na}$ análise multivariada para identificar os fatores de risco para a ocorrência de ao menos um EA relacionado à enfermagem permaneceu no modelo final o tempo de internação maior do que 3 dias (OR simples $=11,44$; OR ajustado $=10,63$; IC $95 \%=6,17-18,31 ; p<0,001)$ e a sobrecarga de trabalho de enfermagem (OR simples $=2,74$; OR ajustado=3,21; IC $95 \%=1,78-5,79 ; p<0,001)$. A análise da proporção de pacientes que sofreram eventos adversos influiu negativamente quanto à perspectiva de sobrevida desses indivíduos, $\mathrm{Chi}^{2}=10,49, \mathrm{p}=0,001$.

Finalmente, a análise univariada para o desfecho óbito nas UTIs incluiu como variáveis independentes: Apache; NAS, idade, tempo de internação nas UTIs, comorbidades e as diferentes categorias de EA. Um modelo foi construído para cada categoria de EA, a saber: geral (qualquer natureza de EA); EA médico, EA relacionado à enfermagem; EA cirúrgico; infecção hospitalar e EA relacionado a medicamentos. A sobrecarga de trabalho de enfermagem (NAS $>51 \%$ ) apresentou associação com óbito dos pacientes nas UTIs pesquisadas com $\mathrm{OR}=11,25$ (IC 95\%=2,70-47,00, $\mathrm{p}=0,001)$.

\section{DISCUSSÃO}

A partir da publicação do relatório "To Err is Human", em 2000, que descreveu que aproximadamente $10 \%$ das internações hospitalares eram complicadas por eventos adversos com consequências para os pacientes e para o sistema de saúde, tornou-se evidente a relevância do tema segurança do paciente, tanto é que a Organização Mundial de Saúde, desde 2004, tem desenvolvido um programa com o propósito de melhorar a segurança do paciente e, por conseguinte reduzir a taxa de $E A s^{(1,9)}$. No documento publicado pela $\mathrm{WHO}$, enfatiza-se que os EAs devem ser pesquisados, desde os fatores que contribuem para sua ocorrência, as características dos pacientes, o tipo de incidente observado, os mecanismos de detecção, os fatores que podem mitigar os EAs, a evolução dos pacientes, o acompanhamento organizacional e as ações de melhoria a ser implementadas e os resultados dessas ações.
No Brasil, o Ministério da Saúde instituiu, em maio de 2013 o Programa Nacional de Segurança do Paciente (PNSP) com o objetivo de implementar medidas assistenciais, educativas e programáticas e de iniciativas voltadas à segurança do paciente em diferentes áreas da atenção, organização e gestão de serviços de saúde, por meio da implantação da gestão de risco e de Núcleos de Segurança do Paciente nos estabelecimentos de saúde ${ }^{(20)}$.

Até o momento, mesmo com esses esforços nacionais e internacionais, a grande maioria das pesquisas corresponde a estudos retrospectivos, baseados em revisões de prontuários ou partir de recuperação de dados a partir de registros eletrônicos, e os resultados encontrados subestimam a real ocorrência de EAs e de incidentes sem lesão, pois nem todas as complicações sofridas pelos pacientes são registradas nos prontuários. Seguramente as taxas reais de ocorrência de incidentes e EAs são ainda mais alarmantes. Rutberg et al., em 2014, demonstraram que apenas $6,3 \%$ dos EAs foram registrados em prontuários espontaneamente, concluindo que estratégias especiais devem ser aplicadas para resultados efetivos ${ }^{(21)}$.

Há que se considerar que alguns EAs são inevitáveis, como a reação alérgica a um antibiótico; mas, entre 37-54\% dos EAs reportados na literatura em pesquisas do tipo retrospectivo são potencialmente evitáveis ${ }^{(2,5,8)}$.

As unidades de terapia intensiva são reconhecidas como setores muito suscetíveis à ocorrência de incidentes e EAs. Alguns aspectos são fundamentais para facilitar a ocorrência de erros nesses locais, como a complexidade dos casos, a necessidade de decisões de alto risco de forma urgente, a falta de informações prévias sobre a saúde dos pacientes, a variabilidade de capacitação dos profissionais médicos e de enfermagem que atuam nesta área, a realização de procedimentos diagnósticos e terapêuticos em frequência muito maior quando comparada a outros setores hospitalares e a utilização de um arsenal medicamentoso muito mais complexo em função da maior gravidade do quadro clínico inicial. Apesar desta vulnerabilidade, poucas são as publicações relacionadas à segurança de pacientes submetidos a cuidados intensivos.

$\mathrm{Na}$ pesquisa aqui apresentada, resultado de um trabalho prospectivo conduzido em duas UTIs de hospitais brasileiros, foram analisados os incidentes sem lesão e os EAs relativos às competências da Enfermagem, o tipo de ocorrência, a evolução dos pacientes, permitindo a identificação e elaboração de ações de melhoria para mitigar essas ocorrências.

Constatamos, inicialmente, a elevada taxa de incidentes sem lesão $(84,6 \%$ ) durante o período estudado e, que $74,2 \%$ das admissões sofreram ao menos um EA durante a permanência nas UTIs avaliadas. Resultados esses superiores ao descrito pela literatura, mas que podem ser, em parte explicados 
pelo fato de ser um estudo prospectivo, em que instrumentos foram especialmente desenvolvidos para esta finalidade, foi feita busca ativa, observação em visitas médicas e da equipe multiprofissional e checagem de exames de imagem e de laboratório postergados e cancelados.

Em pesquisa conduzida em hospital de ensino terciário, avaliou-se a incidência de eventos adversos e incidentes em pacientes de $\mathrm{UT}^{(5)}$. As taxas de eventos adversos e incidentes sem lesão por 1000 pacientes-dia foram respectivamente 80,5 e 149,7 . Dentre os eventos, $13 \%$ destes foram graves ou fatais. Do total de pacientes, $20,2 \%$ sofreram ao menos um evento, uma taxa maior que a de estudos não focados em terapia intensiva. Em outro estudo, observou-se uma incidência maior de eventos adversos em terapia intensiva, que chegou a $39,2 \%{ }^{(10)}$.

$\mathrm{Na}$ pesquisa aqui apresentada encontramos um prolongamento no número de dias de internação nos pacientes que desenvolveram EAs, quando comparados com aqueles que não o apresentaram $(p<0,001)$, corroborando o que já foi descrito na literatura ${ }^{(3,5)}$. Forster et al. ${ }^{(22)}$ identificaram um aumento de 31 dias na média do tempo de permanência de pacientes que sofreram um evento adverso quando internados em terapia intensiva. No nosso estudo, inclusive, foi demonstrado que há uma forte e significativa correlação do tempo e permanência do paciente nas UTIs com o número de EAs de enfermagem.

Observamos que, dentre as ocorrências na competência de enfermagem, predominaram fortemente os incidentes sem lesão $(94,2 \%)$ sobre os EAs e que esses últimos foram considerados evitáveis, ensejando medidas para a mitigação dos mesmos. A identificação de incidentes sem lesão que culminam em evento adverso ou que tem potencial para tal tem utilização prática pois podem direcionar ações educativas, preventivas e corretivas contribuindo para a melhoria da segurança do paciente ${ }^{(23)}$.

No estudo aqui apresentado, utilizou-se um recurso (NAS) que tornou possível demonstrar que, em ambos hospitais participantes, houve sobrecarga de trabalho no período analisado $(p<0,01)$ e que incidentes sem lesão e EAs estavam associados à sobrecarga de trabalho do pessoal de enfermagem $(p<0,0001)$. Na nossa casuística, um enfermeiro cuidou de mais de 2 pacientes em cuidados intensivos, enquanto que a Associação Britânica de Enfermeiros para cuidados críticos recomenda uma proporção de um enfermeiro por paciente em UTI (24).

Cho et al. ${ }^{(25)}$ conduziram um estudo em hospitais norte-americanos que envolveu aproximadamente 10.000 enfermeiros e mais de 230.000 pacientes, tendo concluído que alta proporção de pacientes cirúrgicos por enfermeiro, levou a um maior risco de morte após 30 dias de internação e maior numero de morte por complicações potencialmente evitáveis. Neste trabalho, pudemos demonstrar que a ocorrência de eventos adversos relacionados à Enfermagem levou a um risco de morte significativamente maior $\left(\mathrm{Chi}^{2}=10,49 \mathrm{p}: 0,001\right)$.

$\mathrm{Na}$ nossa pesquisa, admissões que se prolongaram por quatro dias ou mais apresentaram uma chance de desenvolver EAs relacionados à enfermagem de quase 11 vezes em relação às admissões mais curtas. Além deste aspecto, as admissões assistidas por profissionais de enfermagem sobrecarregados tiveram mais do que o dobro de chance de sofrerem ao menos um EA relacionado à enfermagem.
A sobrecarga de trabalho relacionada à desproporção entre o número de profissionais de enfermagem e de pacientes é relatada como fator de risco inclusive para o aumento da incidência de infecções hospitalares em pacientes críticos. Stegenga et al. ${ }^{(26)}$ relataram estudos onde a insuficiência de profissionais de enfermagem foi um fator importante para a ocorrência de infecções hospitalares em unidades de atendimento adulto, neonatal e pediatria. Além de relacionar-se com má adesão às práticas de higiene e antissepsia das mãos pelos profissionais de saúde, a sobrecarga de trabalho é um obstáculo às ações de educação continuada para prevenção contra a infecção hospitalar, onde a disponibilidade para a participação de treinamentos e a eficácia dos mesmos fica prejudicada.

A ocorrência de erros deve ser interpretada como falhas ou não-conformidades decorrentes de colapsos dos complexos sistemas técnicos e organizacionais relacionados à atenção em saúde e não como resultados isolados de ações profissionais. São as vulnerabilidades presentes nos sistemas (fatores latentes) que favorecem a prática de erros pelos indivíduos envolvidos com o cuidado (fatores ativos) ${ }^{(27)}$. Desenvolvem-se, para a sua ocorrência, processos multicausais e de articulação complexa, e é como tais que foram aqui estudados.

Deve-se ressaltar que, frequentemente, o pessoal de enfermagem acumula mais de um emprego, tem alta rotatividade face à baixa remuneração comumente aplicada ou às condições de trabalho na instituição e elevado nível de estresse. Desse modo, as organizações devem prover número adequado de profissionais, bem como condições de trabalho e de remuneração adequados que permitam um menor risco de dano aos pacientes ${ }^{(12)}$. Além do mais, o enfermeiro não tem como função apenas a assistência ao paciente, mas inclui o treinamento e capacitação de profissionais de enfermagem, gerenciamento de insumos e materiais, articulação com outros profissionais da saúde e da administração da organização, orientação dos pacientes e familiares, promovendo, enfim, a gestão multiprofissional em prol do paciente.

Este estudo demonstra o impacto da sobrecarga de trabalho de enfermagem, aumentando os riscos de incidentes sem lesão, de eventos adversos em pacientes em pacientes internados em UTIs em dois hospitais brasileiros, revelando a necessidade de um dimensionamento adequado do quadro de pessoal desta categoria profissional. Deve-se ressaltar que os eventos adversos também se apresentaram com associação significativa para ocorrência de óbito neste estudo. A sobrecarga de trabalho de profissionais de enfermagem deve ser entendida como uma consequência de vários fatores e, para ser solucionada, e cabe ao gestor a empreender estratégias em níveis diversos.

Apesar de sabermos que a gestão de pessoas em âmbito hospitalar é altamente complexa, envolvendo aspectos sistêmicos e também intraorganizacionais, a definição de metas, os recursos disponíveis, as modalidades de contratação de pessoal e remuneração e o tipo de atendimento devem ser analisados de modo global e integrados. Os gestores de recursos humanos devem estar em contato continuado com os coordenadores de enfermagem, procurando obter as informações para diagnosticar a sobrecarga de trabalho de profissionais, uma vez que os coordenadores de enfermagem é que treinam e gerenciam a equipe de enfermagem localmente e 
com essa interação, procurar desenvolver políticas, elaborar estratégias e oferecer o suporte necessário para solucionar essa situação quando detectada. Idealmente, inclusive, a sobrecarga de trabalho deveria ser prevenida, fazendo uma análise detalhada e dinâmica das peculiaridades de cada setor.

Espera-se por meio desse estudo contribuir para uma compreensão mais aprofundada do problema da sobrecarga de trabalho dos profissionais de enfermagem no âmbito da gestão de pessoas na área hospitalar, em especial em unidades de terapia intensiva dos hospitais analisados, tendo por objetivo a melhoria contínua dos serviços prestados em saúde e a redução de eventos adversos relacionados à sobrecarga de trabalho.

\section{CONCLUSÕES}

Esse trabalho, realizado em duas unidades de terapia intensiva de hospitais públicos brasileiros, detectou que aproximadamente $78 \%$ dos incidentes sem lesão e de eventos adversos em pacientes foram relacionados à esfera da Enfermagem. Essas complicações atribuídas à sobrecarga de trabalho de enfermagem aumentaram o número de dias de internação dos pacientes estudados. Demonstrou-se que a sobrecarga de trabalho de enfermagem esteve também associada a um aumento de risco de mortalidade dos pacientes nas UTIs avaliadas. Os eventos adversos relacionados à esfera de enfermagem detectados nesse estudo são passíveis de prevenção pela assistência adequada de enfermagem. É fundamental que os gerentes de enfermagem participem ativamente no processo de gestão de pessoas evitando a sobrecarga de trabalho proporcionando, consequentemente, aumento da segurança do paciente.

\section{AGRADECIMENTOS}

Projeto financiado pela Fapesp 2008/00955-2, a quem agradecemos pela viabilização deste trabalho.

\section{REFERÊNCIAS}

1. Organização Mundial de Saúde. Estrutura concetual da classificação internacional sobre segurança do doente [Internet]. Lisboa: OMS; 2011. [acesso em 03 de junho de 2014]. Disponível em: http://apps.who.int/iris/ bitstream/10665/70882/4/WHO_IER_PSP_2010.2_por. pdf?ua $=1$

2. Nilsson L, Pihl A, Tagsjo M, Ericsson E. Adverse events are common on the intensive care unit: results from a structured record review. Acta Anaesthesiol Scand. 2012;56(8):959-65.

3. Baker GR, Norton PG, Flintoft V, Blais R, Brown A, Cox J, et al. The Canadian adverse events study: the incidence of adverse events among hospital patients in Canada. CMAJ. 2004;170(11):1678-86.

4. Vlayen A, Marquet K, Schrooten W, Vleugels A, Hellings J, Weekers F, et al. Design of a medical record review study on the incidence and preventability of adverse events requiring a higher level of care in Belgian hospitals. BMC Res Notes. 2012;5:468.

5. Rothschild JM, Landrigan CP, Cronin JW, Kaushal R, Lockley SW, Burdick E, et al. The Critical Care Safety Study: the incidence and nature of adverse events and serious medical errors in intensive care. Crit Care Med. 2005;33(8):1694-700.

6. Leape LL, Brennan TA, Laird N, Lawthers AG, Localio AR, Barnes BA, et al. The nature of adverse events in hospitalized patients. Results of the Harvard Medical Practice Study II. N Engl J Med. 1991;324(6):377-84.

7. Daud-Gallotti R, Novaes HMD, Lorenzi MC, Eluf Neto J, Okamura MN, Pizzo VRP. Adverse events in patients with community-acquired pneumonia at an academic tertiary emergency department. Infect Dis Clin Pract. 2006; 14:350-9.

8. Forster AJ, Kyeremanteg K, Hooper J, Shojania KG, van
Walraven C. The impact of adverse events in the intensive care unit on hospital mortality and length of stay. BMC Health Serv Res [Internet]. 2008 [cited 2014 June 03];8:259. Available from: http://www.ncbi.nlm.nih.gov/ pmc/articles/PMC2621200/

9. Kohn LT, Corrigan JM, Donaldson MS, editors. To err is human: building a safer health system. committee on quality in America. Washington (DC): National Academy Press; 2000.

10. Garrouste-Orgeas M, Timsit JF, Vesin A, Schwebel C, Arnodo $P$, Lefrant $J Y$, et al. Selected medical errors in the intensive care unit: results of the IATROREF study: part I and II. Am J Respir Crit Care Med. 2010;181(2):134-42.

11. Kiekkas P, Aretha D, Stefanopoulos N, Baltopoulos GI. Knowledge is power: studying critical incidents in intensive care. Crit Care [Internet]. 2012 [cited 2014 June 03];16(1):102. Available from: http://www.ncbi.nlm.nih. gov/pmc/articles/PMC3396222/

12. Van Bogaert $P$, Timmermans $O$, Weeks $S M$, van Heusden D, Wouters K, Franck E. Nursing unit teams matter: impact of unit-level nurse practice environment, nurse work characteristics, and burnout on nurse reported job outcomes, and quality of care, and patient adverse events-a cross-sectional survey. Int J Nurs Stud. 2014;51(8):1123-34.

13. Carayon P, Gurses AP. A human factors engineering conceptual framework of nursing workload and patient safety in intensive care units. Intensive Crit Care Nurs. 2005;21(5):284-301.

14. Weissman JS, Rothschild JM, Bendavid E, Sprivulis P, Cook EF, Evans RS, et al. Hospital workload and adverse events. Med Care. 2007;45(5):448-55.

15. Charlson ME, Pompei $P$, Ales KL, MacKenzie CR. A new method of classifying prognostic comorbidity in longitudinal studies: development and validation. J Chronic Dis 
[Internet]. 1987 [cited 2014 June 03]40(5):373-83. Available from: http://www.ncbi.nlm.nih.gov/pubmed/3558716

16. Le Gall JR, Lemeshow S, Saulnier F. A new Simplified Acute Physiology Score (SAPS II) based on a European/North American multicenter study. JAMA. 1993;270(24):2957-63.

17. Miranda DR, Nap R, Rijk A, Schaufeli W, lapichino G; TISS Working Group. Therapeutic Intervention Scoring System. Nursing activities score. Crit Care Med [Internet]. 2003 [cited 2014 June 03];31(2):374-82. Available from: http://www.ncbi.nlm.nih.gov/pubmed/12576939

18. Queijo AF, Padilha KG. Instrumento de medida da carga de trabalho de enfermagem em Unidade de Terapia Intensiva: Nursing Activities Score (N.A.S). Rev Paulista Enf. 2004;23(2):114-122.

19. Daud-Gallotti RM, Costa SF, Guimarães T, Padilha KG, Inoue EN, Vasconcelos TN, et al. Nursing Workload as a Risk Factor for Healthcare Associated Infections in ICU: a prospective study. PLoS ONE [Internet]. 2012 [cited 2014 June 03];7(12):e52342. Available from: http://www.hcnet. usp.br/humaniza/pdf/Nursing \% 20Workload \% 20as $\% 20$ a $\% 20$ Risk $\% 20$ Factor $\% 20$ for $\% 20$ Healthcare $\% 20$ Associated $\% 20$ Infections $\% 20 \mathrm{in} \% 20 \mathrm{ICU}$.pdf

20. Ministério da Saúde (BR). Portaria $n^{\circ}$. 529, de $1^{\circ}$ de abril de 2013. Institui o Programa Nacional de Segurança do Paciente [Internet]. Diário Oficial da União 2013 [acesso em 12 de junho de 2014]. Disponível em: http://bvsms.saude.gov.br/bvs/saudelegis/gm/2013/ prt0529_01_04_2013.htmll
21. Rutberg H, Risberg MB, Sjodahl R, Nordqvist $P$, Valter $L$, Nilsson L. Characterisations of adverse events detected in a university hospital: a 4-year study using the Global Trigger Tool method. BMJ Open [Internet]. 2014 [cited 2014 June 03];4(5):e004879. Available from: http://psnet.ahrq. gov/resource.aspx? resourcelD $=28024$

22. Forster AJ, Rose NG, van Walraven C, Stiell I. Adverse events following an emergency department visit. Qual Saf Health Care. 2007;16(1):17-22.

23. Kiekkas P, Aretha D, Stefanopoulos N, Baltopoulos GI. Knowledge is power: studying critical incidents in intensive care. Crit Care [Internet]. 2012 [cited 2014 June 03];16(1):102. Available from: http://bvsalud.org/portal/ resource/en/mdl-22236387

24. Bray K, Wren I, Baldwin A, St Ledger U, Gibson V, Goodman $S$, et al. Standards for nurse staffing in critical care units determined by: the British Association of Critical Care Nurses, The Critical Care Networks National Nurse Leads, Royal College of Nursing Critical Care and In-flight Forum. Nurs Crit Care. 2010;15(3):109-111.

25. Cho SH, Ketefian S, Barkauskas VH, Smith DG. The effects of nurse staffing on adverse events, morbidity, mortality, and medical costs. Nurs Res. 2003;52(2):71-9.

26. Stegenga J, Bell E, Matlow A. The role of nurse understaffing in nosocomial viral gastrointestinal infections on a general pediatrics ward. Infect Control Hosp Epidemiol. 2002;23(3):133-6.

27. Leape LL, Berwick DM. Five years after To Err Is Human: what have we learned? JAMA. 2005 18;293(19):2384-90. 